\title{
Patient comfort during flexible and rigid cystourethroscopy
}

\author{
Wojciech Krajewski ${ }^{1}$, Romuald Zdrojowy ${ }^{1}$, Joanna Wojciechowska ${ }^{2}$, Katarzyna Kościelska ${ }^{3}$, Janusz Dembowski ${ }^{1}$, \\ Michał Matuszewski ${ }^{1}$, Krzysztof Tupikowski ${ }^{1}$, Bartosz Małkiewicz ${ }^{1}$, Anna Kołodziej ${ }^{1}$ \\ ${ }^{1}$ Department of Urology and Oncological Urology, Wroclaw Medical University, Wroclaw, Poland \\ ${ }^{2}$ Department of Otolaryngology and Head and Neck Surgery, Wroclaw Medical University, Wroclaw, Poland \\ ${ }^{3}$ Department of Nephrology and Transplantation Medicine, Wroclaw Medical University, Wroclaw, Poland
}

Videosurgery Miniinv 2016; 11 (2): 94-97 DOI: $10.5114 /$ wiitm.2016.60665

\begin{abstract}
Introduction: Cystourethroscopy (CS) is an endoscopic method used to visualize the urethra and the bladder. Aim: In this study, we prospectively evaluated pain in men undergoing cyclic cystoscopic assessment with rigid and flexible instruments after transurethral resection of bladder tumor (TURB).

Material and methods: One hundred and twenty male patients who were under surveillance after a TURB procedure due to urothelial cell carcinoma and who had undergone at least one rigid cystourethroscopy in the past were enrolled in the trial. Patients were prospectively randomized to age-matched groups for flexible (group F) or rigid (group R) CS. Patient's comfort was evaluated on an 11-grade scale, ranging from 0 (free from pain) to 10 points (unbearable pain). Results: The patients described the pain during the previous rigid CS as ranging from 4 to 10 (mean: 6.8) in group $F$ and from 0 to 10 (mean: 5.8) in group R. Group R patients described the pain during the current rigid CS as ranging from 0 to 10 (mean: 5.7). No mean change in the grade was observed between the two pain descriptions (no change 11 patients, weaker pain 25 patients, stronger pain 24 patients, gamma 0.51, $p<0.0001$ ). Group F described the pain as 1 to 5 (mean: 2.1). In the case of flexible CS the pain experience was greatly lowered compared to the previous rigid CS. All flexible CS patients reported lowered pain (by 1 to 9 grades). Patients' age did not influence the comfort of the flexible CS or the change in pain level.

Conclusions: Flexible CS is better tolerated than rigid cystoscopy by male patients regardless of patients' age.
\end{abstract}

Key words: rigid cystoscopy, flexible cystoscopy, pain.

\section{Introduction}

Cystourethroscopy (CS) is an endoscopic method used to visualize the urethra and the bladder. It is commonly used by urologists for evaluation of hematuria, voiding symptoms, performance of minor procedures such as foreign body removal and surveillance of urothelial carcinoma. Cystourethroscopy may be performed using either rigid or flexible cystourethroscopes, and both rigid and flexible devices were shown to have equal efficacy in identifying tumors in the bladder [1, 2].
Compared with flexible endoscopes, rigid instruments offer better image quality, a wider lumen of the working channel, improved irrigant flow and superior handiness. However, flexible cystourethroscopes provide more options for patient positioning, enable smooth passage over an elevated bladder neck or median lobe, facilitate full inspection of the bladder because of their movable tip and, what is most important, significantly improve patient comfort.

Only a few old and two recent studies comparing patient comfort are available; however, they are

\section{Address for correspondence}

Wojciech Krajewski, Department of Urology and Oncological Urology, Wroclaw Medical University, 213 Borowska St, 50-556 Wroclaw, Poland, phone: +48 691510 609, e-mail: wk@softstar.pl 
based on heterogeneous groups of patients and/or disorders, and some of them have unclear methodology or include CS in general anesthesia, which is no longer routinely performed nowadays [3-6].

There are not enough objective data on patient comfort in the literature to demonstrate authoritatively superiority of either of these methods.

\section{Aim}

In this study, we prospectively evaluated pain in men undergoing cyclic cystoscopic assessment with rigid and flexible instruments after transurethral resection of bladder tumor (TURB).

\section{Material and methods}

Appropriate institutional ethics committee approved the study.

We evaluated pain perception of male patients who had undergone at least one rigid cystourethroscopy procedure in our department as standard surveillance after a TURB procedure due to urothelial cell carcinoma (UCC) and were scheduled for the next CS procedure.

From the group of 120 patients, 60 patients were randomly assigned to have current flexible CS (group F), and the other 60 patients underwent current rigid CS as controls (group $\mathrm{R}$ ).

All procedures were performed in the Urology and Urologic Oncology Department of Wroctaw Medical University, and one urologist conducted all flexible procedures.

Exclusion criteria included CS with any type of intervention, patients with ureteral catheters, history of any but TURB surgery on the genitourinary tract and urinary tract infection.

Patient's comfort was evaluated with a numeric rating scales (NRS) [7]. An 11-point numerical scale, ranging from 0 (free from pain) to 10 points (unbearable pain) was used. Comfort was estimated a few minutes after every CS.

Procedures were performed with a rigid Storz 20 French and flexible 15 French Wolf cystourethroscope.

All instruments were inserted and advanced under direct operator vision.

All procedures started with standard disinfection of external genitalia with an antiseptic agent. Injection of a lubricant containing $2 \%$ lidocaine was performed at least 5 min before instrument insertion.
As is well known, this maneuver reduces pain and enhances male patient comfort [8]. All actions were performed without any systemic sedation or analgesia. No antimicrobial prophylaxis was used routinely. All CS procedures were carried out in the dorsal lithotomy position.

\section{Statistical analysis}

Statistical analysis was performed using Statistica 12.0 (StatSoft, Poland). The distribution changes were analyzed with the $\chi^{2}$ test. The correlations between scores and age were performed with Spearman correlation and comparison of two score results with $\gamma$ statistics.

\section{Results}

The patients described the pain during the previous rigid CS as ranging from 4 to 10 (mean 6.8) in group $\mathrm{F}$ and from 0 to 10 (mean: 5.8 ) in group $\mathrm{R}$ (Figure 1). No influence of age on the pain experience was observed ( $r s=-0.062, p=0.50$ ). Group $R$ patients described the pain during the current rigid CS as ranging from 0 to 10 (mean: 5.7). No mean change in the grade was observed between the two pain descriptions (no change 11 patients, weaker pain 25 patients, stronger pain 24 patients, $\gamma=0.51$, $p<0.0001)$. Again, after the $2^{\text {nd }}$ rigid CS no influence of age was observed ( $r s=-0.024, p=0.85$ ). Group $F$ described the pain as 1 to 5 (mean: 2.1). In the case of flexible CS the pain experience was greatly lowered compared to the previous rigid CS (Figure 1, $p<0.0001$ ). All flexible CS patients reported lowered pain (by 1 to 9 grades). Patients' age did not influence the reported comfort of the flexible CS ( $r s=0.046$, $p=0.73$ ) or the change in the pain level (Figure 2).

\section{Discussion}

To date only two studies with reliable methodology evaluating patients' comfort during CS have been conducted. In the EAU guidelines there are no recommendations suggesting any of the techniques as more favorable. There are no evidence-based data indicating which type of CS is better for a given patient and what the risk factors of elevated pain perception during the procedure are.

We analyzed a homogeneous group of 120 men who were under cystoscopic surveillance after TURB treatment because of UCC. We concluded that rigid 


\begin{tabular}{|c|c|c|c|c|}
\hline \multirow[t]{2}{*}{ Pain } & \multicolumn{2}{|c|}{ Group F } & \multicolumn{2}{|c|}{ Group R } \\
\hline & $1^{\text {st }}$ CS (rigid) & $2^{\text {nd }}$ CS (flex) & $1^{\text {st }}$ CS (rigid) & $2^{\text {nd }} \mathrm{CS}$ (rigid) \\
\hline 0 & & & 1[ & 27 \\
\hline 1 & & 19 & 1 & 3 \\
\hline 2 & & 23 & 2 & 5 \\
\hline 3 & & 12 & 2 & 3 \\
\hline 4 & $3 \square$ & $3 \square$ & 7 & 7 \\
\hline 5 & 14 & $3 \square$ & 21 & 7 \\
\hline 6 & 13 & & 6 & 9 \\
\hline 7 & 11 & & 6 & 7 \\
\hline 8 & 7 & & 7 & 8 \\
\hline 9 & 5 & & $2 \square$ & $2 \square$ \\
\hline 10 & 7 & & 5 & 7 \\
\hline
\end{tabular}

Figure 1. Distribution of pain descriptions after $1^{\text {st }}$ and $2^{\text {nd }} \mathrm{CS}$ in both study groups. The number of patients describing the pain as a given grade is presented on the left

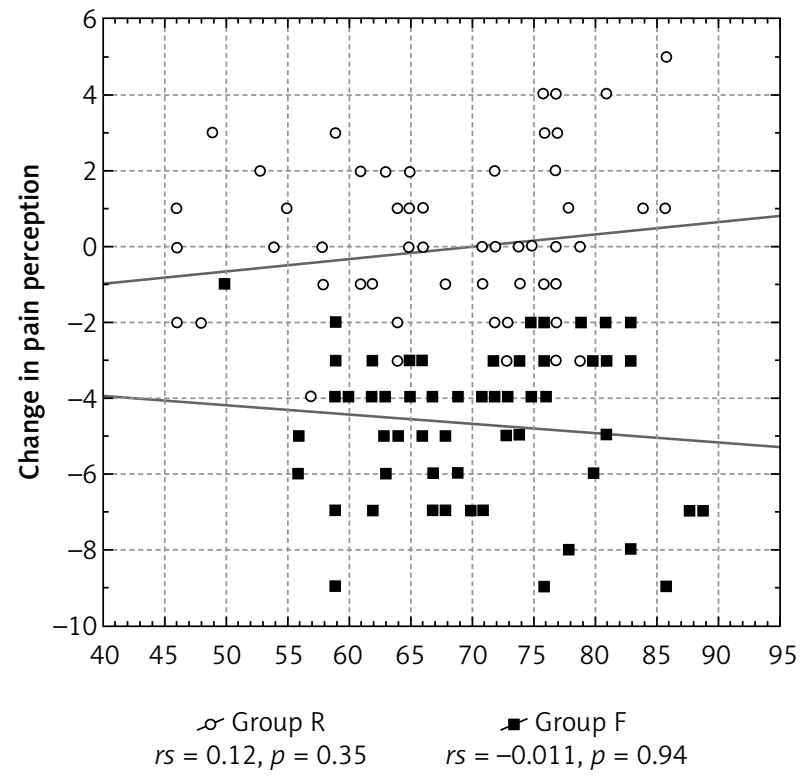

Figure 2. Scatterplot showing no relation of a change in pain perception between $2^{\text {nd }}$ and $1^{\text {st }}$ CS to patients' age

CS was associated with significantly greater pain experienced by the patient during the procedure. Additionally, similar to the results of Seklehner et al., rigid CS was often associated with severe or even unbearable pain [5].

However, we observed that there was no statistically significant difference in pain perception be- tween younger and older patients, which is inconsistent with Seklehner's findings. Rather we can conclude that patients have a given susceptibility to pain perception, which in the case of repeated rigid CS manifested in correlated pain scores $(\gamma=0.51$, $p<0.0001)$. In the case of group F patients, who reported largely decreased pain scores after the $2^{\text {nd }} \mathrm{CS}$, no correlation between feelings was noted $(\gamma=0.10$, $p=0.37)$.

As the pain perception during rigid CS seems to be a characteristic of a given patient, and it is largely decreased when flexible CS is performed, we believe that risk factors should be defined to identify patients who are particularly sensitive to pain. These patients should be referred to a hospital in which it is possible to perform flexible CS, because the fear of pain can be a sufficient reason to abandon the periodic surveillance after surgical treatment of UCC. For those patients rigid CS under general anesthesia is an option, but it is related to higher cost and higher risk of complications and cannot be performed in an outpatient department.

We chose not to assess pain several days after the procedure, because it was proven that patient comfort was similar in both groups at a later time after CS [5].

This study has some limitations. Firstly, rigid cystoscopy was not conducted by one surgeon. Secondly, the studied group was small; however, it was homogeneous. 


\section{Conclusions}

Flexible CS is better tolerated than rigid cystoscopy by male patients independently of patients' age.

\section{Conflict of interest}

The authors declare no conflict of interest.

\section{References}

1. Clayman RV, Reddy P, Lange PH. Flexible fiberoptic and rigid-rod lens endoscopy of the lower urinary tract: a prospective controlled comparison. J Urol 1984; 131: 715-6.

2. Walker L, Liston TG, Lloyd-Davies RW. Does flexible cystoscopy miss more tumours than rod-lens examination? Br J Urol 1993; 72: 449-50.

3. Flannigan GM, Gelister JS, Noble JG, et al. Rigid versus flexible cystoscopy. A controlled trial of patient tolerance. $\mathrm{Br} J$ Urol 1988; 62: 537-40.

4. Denholm SW, Conn IG, Newsam JE, et al. Morbidity following cystoscopy: comparison of flexible and rigid techniques. $\mathrm{Br}$ J Urol 1990; 66: 152-4.

5. Seklehner S, Remzi M, Fajkovic H, et al. Prospective multi-institutional study analyzing pain perception of flexible and rigid cystoscopy in men. Urology 2015; 85: 737-41.

6. Greenstein A, Greenstein I, Senderovich S, et al. Is diagnostic cystoscopy painful? Analysis of 1,320 consecutive procedures. Int Braz J Urol 2014; 40: 533-8.

7. Hjermstad MJ, Fayers PM, Haugen DF, et al. Studies comparing Numerical Rating Scales, Verbal Rating Scales, and Visual Analogue Scales for assessment of pain intensity in adults: a systematic literature review. J Pain Symptom Manage 2011; 41: 1073-93.

8. Aaronson DS, Walsh TJ, Smith JF, et al. Meta-analysis: does lidocaine gel before flexible cystoscopy provide pain relief? BJU Int 2009; 104: 506-9; discussion 9-10.

Received: 22.03.2016, accepted: 12.05.2016. 